\title{
Some identities of generalized Tribonacci and Jacobsthal polynomials
}

\author{
Abdeldjabar Hamdi ${ }^{1}$ and Salim Badidja ${ }^{2}$ \\ ${ }^{1}$ Faculty of Mathematics, University of Youcef Benkhedda Algiers 01 \\ 02 Rue Didouche Mourad, 16000 Algeria \\ e-mail: h. abdeldjabar@gmail.com \\ ${ }^{2}$ Faculty of Mathematics, University of Kasdi Merbah \\ Ouargla Route de Ghardaia, BP. 511, 30000 Algeria \\ e-mail: badidja@hotmail.fr
}

\begin{abstract}
In this study, we denote $\left(t_{n}^{\prime}(x)\right)_{n \in \mathbb{N}}$ the generalized Tribonacci polynomials, which are defined by $t_{n}^{\prime}(x)=x^{2} t_{n-1}^{\prime}(x)+x t_{n-2}^{\prime}(x)+t_{n-3}^{\prime}(x), n \geqslant 4$, with $t_{1}(x)=a, t_{2}(x)=b$, $t_{3}(x)=c x^{2}$ and we drive an explicit formula of $\left(t_{n}^{\prime}(x)\right)_{n \in \mathbb{N}}$ in terms of their coefficients $T^{\prime}(n, j)$, Also, we establish some properties of $\left(t_{n}(x)\right)_{n \in \mathbb{N}}$. Similarly, we study the Jacobsthal polynomials $\left(J_{n}(x)\right)_{n \in \mathbb{N}}$, where $J_{n}(x)=J_{n-1}(x)+x J_{n-2}(x)+x^{2} J_{n-3}(x), n \geqslant 4$, with $J_{1}(x)=J_{2}(x)=1$, $J_{3}(x)=x+1$ and describe some properties.
\end{abstract}

Keywords: Tribonacci polynomials, Generalized Tribonacci polynomials, Jacobsthal polynomials. 2020 Mathematics Subject Classification: 11B39, 11 B83.

\section{Introduction}

Recently, Tribonacci and Jacobsthal sequences have investigated very largely and authors tried to developed and give some directions to mathematical calculations using these type of special sequences. One of these directions goes through to the Tribonacci and the Jacobsthal polynomials. In fact, Tribonacci sequences have been firstly defined by M. Feinberg in 1963 and then some important properties. On the other hand, in 1973, Hoggatt and Bicknell defined the Tribonacci polynomials $t_{n}(x)$.

There are many studies on Tribonacci polynomial and its properties (see [1,4,5]). Also it has been generalized in many ways (see [7]). 
The first main idea in this paper is to study the generalized Tribonacci polynomials $t_{n}^{\prime}(x)$.

After this, we show the link between $t_{n}^{\prime}(x)$ and $t_{n}(x)$ by using the $Q$-matrix. We derive an explicit formula for the generalized Tribonacci polynomials with the element of array $T^{\prime}(n, j)$ (see Definition 3.2).

Finally in the analogous sense, we investigate everything that was previously done (on Tribonacci polynomials) for the other kind of polynomials called Jacobsthal polynomials $J_{n}(x)$. Hence, we establish some properties of the Jacobsthal polynomials $J_{n}(x)$

\section{Preliminaries}

Definition 2.1 ([5]). The Tribonacci polynomials $t_{n}(x)$ are defined by

$$
\left\{\begin{array}{l}
t_{n}(x)=x^{2} t_{n-1}(x)+x t_{n-2}(x)+t_{n-3}(x), \\
t_{0}(x)=0, t_{1}(x)=1, t_{2}(x)=x^{2}
\end{array}\right.
$$

In [5], Tribonacci array is defined in the following way: Let $T(n, j)$ denote the element in row $n$ and column $j$ of this array, where $n>j>0$. It satisfies the recurrence relation

$$
T(n, j)=T(n-1, j)+T(n-2, j-1)+T(n-3, j-2)
$$

where $n>4$. The following table shows the first elements.

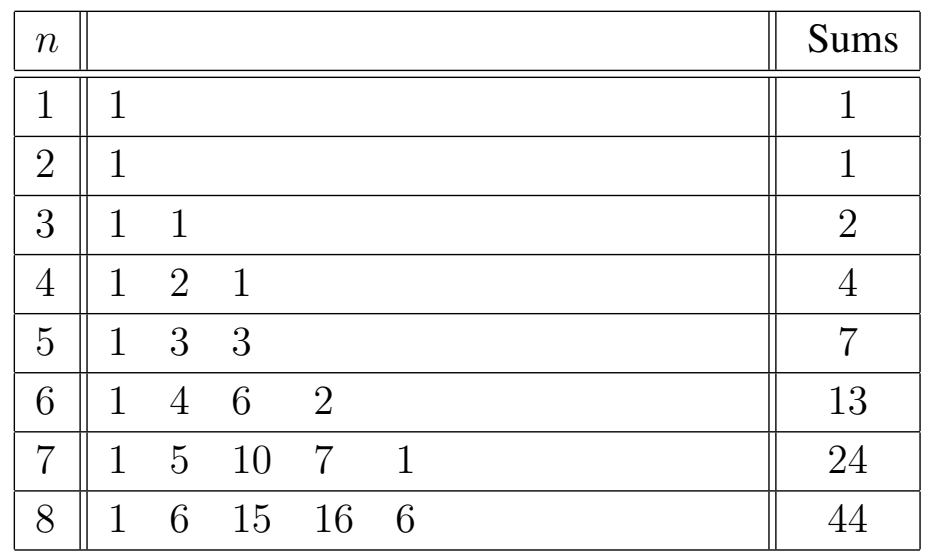

Table 1. Tribonacci coefficients.

Using the elements of the Tribonacci array $T(n, j)$, Tribonacci polynomial $t_{n}(x)$ is defined [5] by the formula

$$
t_{n}(x)=\sum_{j=0}^{[(2 n-2) / 3]} T(n, j) x^{2 n-3 j-2}
$$

Definition 2.2 ([1]). The generalized Tribonacci sequence $\left(T_{n}^{\prime}\right)_{n \in \mathbb{N}}$ is defined by the recurrence linear relation

$$
\left\{\begin{array}{l}
T_{n}^{\prime}=T_{n-1}^{\prime}+T_{n-2}^{\prime}+T_{n-3}^{\prime}, n \geq 4 \\
T_{1}^{\prime}=a, T_{2}^{\prime}=b, T_{3}^{\prime}=c, \text { for } a, b \text { and } c \in \mathbb{Z}
\end{array} .\right.
$$


Theorem 2.1 ([8]). The Tribonacci numbers $T_{n}$ can be expressed by using Binet's formula, we get

$$
T_{n}=\frac{a^{n+1}}{(\alpha-\beta)(\alpha-\gamma)}+\frac{\beta^{n+1}}{(\beta-\alpha)(\beta-\gamma)}+\frac{\gamma^{n+1}}{(\gamma-\alpha)(\gamma-\beta)},
$$

where $\alpha, \beta$ and $\gamma$ are the distinct roots of the cubic equation $x^{3}-x^{2}-x-1=0$,

Theorem 2.2 ([7]). The $n$-th Tribonacci polynomial is given by

$$
t_{n}(x)=\frac{\mathfrak{R}_{1}^{n+1}}{\left(\mathfrak{R}_{1}-\mathfrak{R}_{2}\right)\left(\mathfrak{R}_{1}-\mathfrak{R}_{3}\right)}+\frac{\mathfrak{R}_{2}^{n+1}}{\left(\mathfrak{R}_{2}-\mathfrak{R}_{1}\right)\left(\mathfrak{R}_{2}-\mathfrak{R}_{3}\right)}+\frac{\mathfrak{R}_{3}^{n+1}}{\left(\mathfrak{R}_{3}-\mathfrak{R}_{1}\right)\left(\mathfrak{R}_{3}-\mathfrak{R}_{2}\right)},
$$

where $\mathfrak{R}_{1}, \mathfrak{R}_{2}$ and $\mathfrak{R}_{3}$ are the distinct roots of the characteristic polynomial of (1)

$$
t^{3}-x^{2} t^{2}-x t-1=0
$$

Definition 2.3 ([7]). The Tricobsthal polynomials are defined by the recurrence linear relation

$$
\left\{\begin{array}{l}
J_{n}(x)=J_{n-1}(x)+x J_{n-2}(x)+x^{2} J_{n-3}(x), \text { for } n \geqslant 4 \\
J_{1}(x)=J_{2}(x)=1, J_{3}(x)=x+1
\end{array} .\right.
$$

If we take $x=1$, then $J_{n}(1)=t_{n}(1)=T_{n}$ becomes the famous Tribonacci sequence .

Analogously, we can define generalized Tricobsthal polynomials as follows

Definition 2.4. The generalized Tricobsthal polynomials are defined by recurrence linear relation, given by

$$
\left\{\begin{array}{l}
J_{n}^{\prime}(x)=J_{n-1}^{\prime}(x)+x J_{n-2}^{\prime}(x)+x^{2} J_{n-3}^{\prime}(x), \text { for } n \geqslant 4 \\
J_{1}^{\prime}(x)=a, J_{2}^{\prime}(x)=b, J_{3}^{\prime}(x)=c_{1} x+c_{0}
\end{array}\right.
$$

where parameters $c_{1}$ are positive integers and $a, b, c_{0}$ are non-negative integers.

When $a=b=c_{1}=c_{0}=1$, then one gets Tricobsthal polynomials introduced earlier.

Theorem 2.3. The n-th Tricobsthal polynomials is given by

$$
j_{n}(x)=\frac{\left(\mathfrak{R}_{1}^{\prime}\right)^{n+1}}{\left(\mathfrak{R}_{1}^{\prime}-\mathfrak{R}_{2}\right)\left(\mathfrak{R}_{1}^{\prime}-\mathfrak{R}_{3}^{\prime}\right)}+\frac{\left(\mathfrak{R}_{2}^{\prime}\right)^{n+1}}{\left(\mathfrak{R}_{2}^{\prime}-\mathfrak{R}_{1}^{\prime}\right)\left(\mathfrak{R}_{2}^{\prime}-\mathfrak{R}_{3}^{\prime}\right)}+\frac{\left(\mathfrak{R}_{3}^{\prime}\right)^{n+1}}{\left(\mathfrak{R}_{3}^{\prime}-\mathfrak{R}_{1}^{\prime}\right)\left(\mathfrak{R}_{3}^{\prime}-\mathfrak{R}_{2}^{\prime}\right)},
$$

where $\mathfrak{R}_{1}^{\prime}, \mathfrak{R}_{2}^{\prime}$ and $\mathfrak{R}_{3}^{\prime}$ are the distinct roots of the characteristic polynomials of (6)

$$
t^{3}-t^{2}-x t-x^{2}=0
$$

\section{Main results}

\subsection{Properties of generalized Tribonacci polynomials}

Definition 3.1. The generalized Tribonacci polynomials $t_{n}^{\prime}(x)$, are defined by recurrence linear relation

$$
\left\{\begin{array}{l}
t_{n}^{\prime}(x)=x^{2} t_{n-1}^{\prime}(x)+x t_{n-2}^{\prime}(x)+t_{n-3}^{\prime}(x), n \geqslant 4 \\
t_{1}^{\prime}(x)=a, t_{2}^{\prime}(x)=b, t_{3}^{\prime}(x)=c x^{2}
\end{array} .\right.
$$

Theorem 3.1. For $n>4$, we have

$$
t_{n}^{\prime}(x)=c t_{n-2}(x)+b\left[x t_{n-3}(x)+t_{n-4}(x)\right]+a t_{n-3}(x) .
$$


Proof. We define the square matrix $Q$ of order 3 as:

$$
Q=\left[\begin{array}{ccc}
x^{2} & x & 1 \\
1 & 0 & 0 \\
0 & 1 & 0
\end{array}\right]
$$

such that $\operatorname{det} Q=1, Q$ is called the generating matrix for the recurrence relation (10). So

$$
\left(\begin{array}{c}
t_{n+3}^{\prime}(x) \\
t_{n+2}^{\prime}(x) \\
t_{n+1}^{\prime}(x)
\end{array}\right)=\left(\begin{array}{ccc}
x^{2} & x & 1 \\
1 & 0 & 0 \\
0 & 1 & 0
\end{array}\right)^{n}\left(\begin{array}{c}
t_{3}^{\prime}(x) \\
t_{2}^{\prime}(x) \\
t_{1}^{\prime}(x)
\end{array}\right)
$$

it is well known [5] that

$$
\left(\begin{array}{ccc}
x^{2} & 1 & 0 \\
x & 0 & 1 \\
1 & 0 & 0
\end{array}\right)^{n}=\left(\begin{array}{ccc}
t_{n+1}(x) & t_{n}(x) & t_{n-1}(x) \\
x t_{n}(x)+t_{n-1}(x) & x t_{n-1}(x)+t_{n-2}(x) & x t_{n-2}(x)+t_{n-3}(x) \\
t_{n}(x) & t_{n-1}(x) & t_{n-2}(x)
\end{array}\right),
$$

which implies that

$$
\left(\begin{array}{c}
t_{n+3}^{\prime}(x) \\
t_{n+2}^{\prime}(x) \\
t_{n+1}^{\prime}(x)
\end{array}\right)=\left(\begin{array}{ccc}
t_{n+1}(x) & x t_{n}(x)+t_{n-1}(x) & t_{n}(x) \\
t_{n}(x) & x t_{n-1}(x)+t_{n-2}(x) & t_{n-1}(x) \\
t_{n-1}(x) & x t_{n-2}(x)+t_{n-3}(x) & t_{n-2}(x)
\end{array}\right)\left(\begin{array}{c}
t_{3}^{\prime}(x) \\
t_{2}^{\prime}(x) \\
t_{1}^{\prime}(x)
\end{array}\right)
$$

Then

$$
t_{n}^{\prime}(x)=c x^{2} t_{n-2}(x)+b\left[x t_{n-3}(x)+t_{n-4}(x)\right]+a t_{n-3}(x) .
$$

Remark 3.1. If $x=1$, we have

$$
T_{n}^{\prime}=c T_{n-2}+b\left[T_{n-3}+T_{n-4}\right]+a T_{n-3} .
$$

Proposition 3.1. For every integer $n \geq 6$, we have $t_{n}^{\prime}(x)=t_{1}^{\prime}(x)+t_{2}^{\prime}(x)+t_{3}^{\prime}(x)+\left(x^{2}+x\right) \sum_{k=3}^{n-3} t_{k}^{\prime}(x)+\left(x^{2}-1\right)\left(t_{n-1}^{\prime}(x)+t_{n-2}^{\prime}(x)\right)+x t_{n-2}^{\prime}(x)$.

Remark 3.2. The Tribonacci polynomials $\left\{t_{n}(x)\right\}_{n \geq 0}$ can be extended to negative subscripts by defining:

$$
\left\{\begin{array}{c}
t_{-n}(x)=t_{-(n-3)}(x)-x^{2} t_{-(n-2)}(x)-x t_{-(n-1)}(x), n \geq 3 \\
t_{0}(x)=t_{-1}(x)=0, t_{-2}(x)=1
\end{array} .\right.
$$

Proposition 3.2. For $n \geq 3$, we have

1) $t_{-n}(x)=\left(1+x^{3}\right) t_{-n+3}(x)-x t_{-n+4}(x)$

2) $t_{-n}(x)=\left[t_{n-1}(x)\right]^{2}-t_{n}(x) t_{n-2}(x)$

Proof. 1) Proved by using the recurrence relation (12).

2) It is well known [5] that

$$
Q=\left[\begin{array}{ccc}
x^{2} & x & 1 \\
1 & 0 & 0 \\
0 & 1 & 0
\end{array}\right]
$$

such that $\operatorname{det}(Q)=1$, and $Q^{-n}=\left(Q^{n}\right)^{-1}$, it follows that 


$$
Q^{-n}=\left(\begin{array}{lll}
t_{-n+1}(x) & x t_{-n}(x)+t_{-n-1}(x) & t_{-n}(x) \\
t_{-n}(x) & x t_{-n-1}(x)+t_{-n-2}(x) & t_{-n-1}(x) \\
t_{-n-1}(x) & x t_{-n-2}(x)+t_{-n-3}(x) & t_{-n-2}(x)
\end{array}\right)
$$

and

$$
\left(Q^{n}\right)^{-1}=\left(\begin{array}{lrr}
{\left[t_{n-2}(x)\right]^{2}-t_{n-1}(x) t_{n-3}(x)} & t_{n}(x) t_{n-3}(x)-t_{n-1}(x) t_{n-2}(x) & {\left[t_{n-1}(x)\right]^{2}-t_{n}(x) t_{n-2}(x)} \\
{\left[t_{n-1}(x)\right]^{2}-t_{n}(x) t_{n-2}(x)} & t_{n+1}(x) t_{n-2}(x)-t_{n}(x) t_{n-1}(x) & {\left[t_{n}(x)\right]^{2}-t_{n+1}(x) t_{n-1}(x)} \\
{\left[t_{n}(x)\right]^{2}-t_{n+1}(x) t_{n-1}(x)} & t_{n+2}(x) t_{n-1}(x)-t_{n+1}(x) t_{n}(x) & {\left[t_{n+1}(x)\right]^{2}-t_{n+2}(x) t_{n}(x)}
\end{array}\right)
$$

Then

$$
t_{-n}(x)=\left[t_{n-1}(x)\right]^{2}-t_{n}(x) t_{n-2}(x)
$$

\subsection{Generalized Tribonacci array}

Definition 3.2. Let $T^{\prime}(n, j)$ denote the element in row $n$ and column $j$ of the generalized Tribonacci array. It satisfies the recurrence relation

$$
\left\{\begin{array}{rl}
T^{\prime}(n, j) & =T^{\prime}(n-1, j)+T^{\prime}(n-2, j-2)+T^{\prime}(n-3, j-4) \\
T^{\prime}(n+3,3) & =T^{\prime}(n, 0)+T^{\prime}(n+1,1)+T^{\prime}(n+2,3) \\
T^{\prime}(n+2,4) & =T^{\prime}(n+1,4)+T^{\prime}(n, 2)
\end{array},\right.
$$

\begin{tabular}{|c|c|c|c|c|c|}
\hline$n$ & & & & & Sums \\
\hline 1 & $a$ & & & & $a$ \\
\hline 2 & $b$ & & & & $b$ \\
\hline 3 & $c$ & & & & $c$ \\
\hline 4 & $c \quad b$ & $a$ & & & $a+b+c$ \\
\hline 5 & $c \quad b+c$ & $a b$ & & & $a+2 b+2 c$ \\
\hline 6 & $c \quad b+2 c$ & $a \quad 2 b+c$ & $\mathrm{a}$ & & $2 a+3 b+4 c$ \\
\hline 7 & $c \quad b+3 c$ & $a 3 b+3 c$ & $2 a \quad 2 b$ & $a$ & $4 a+6 b+7 c$ \\
\hline 8 & $c \quad b+4 c$ & $a \quad 4 b+6 c$ & $3 a \quad 5 b+2 c$ & $3 a \quad b$ & $7 a+11 b+13 c$ \\
\hline 9 & $c \quad b+5 c$ & $a \quad 5 b+10 c$ & $4 a 9 b+7 c$ & $6 a \quad 5 b+c \quad 2 a$ & $13 a+20 b+24 c$ \\
\hline
\end{tabular}

where $T^{\prime}(n, 0)=c, T^{\prime}(n, 2)=a, n \geqslant 6$. The following table shows the first elements.

Table 2. Generalized Tribonacci coefficients.

\subsection{Generalized Tribonacci formula}

Theorem 3.2. Using the elements of the generalized Tribonacci array $T^{\prime}(n, j)$ to define the generalized Tribonacci polynomial $t_{n}^{\prime}(x)$, by the formula

$$
t_{n}^{\prime}(x)=T^{\prime}(n, 0) x^{2 n-4}+\sum_{j=0}^{[(2 n-7+i) / 3]} T^{\prime}(n, 2 j+1) x^{2 n-3 j-7}+\sum_{j=1}^{[(2 n-4+k) / 3]} T^{\prime}(n, 2 j) x^{2 n-3 j-5}
$$

where $n \equiv i[3], 0 \leqslant i \leqslant 2$, and $k=\frac{(-1)^{i+1} i^{2}+i}{2}$. 
Proof. We distinguish three cases $n \equiv 0[3], n \equiv 1[3]$ and $n \equiv 2[3]$.

Firstly, $n \equiv 0[3]$.

By induction on $n$, it is clear the result is true for $n=6$ and $n=9$ by hypothesis. Assume that it is true for $i$ such that $0 \leq i \leq r+2$, then

$$
t_{r}^{\prime}(x)=T^{\prime}(r, 0) x^{2 r-4}+\sum_{j=0}^{(2 r-9) / 3} T^{\prime}(r, 2 j+1) x^{2 r-3 j-7}+\sum_{j=1}^{(2 r-6) / 3} T^{\prime}(r, 2 j) x^{2 r-3 j-5}
$$

and

$$
\begin{gathered}
x^{2} t_{r+2}^{\prime}(x)=T^{\prime}(r+2,0) x^{2 r+2}+\sum_{j=0}^{(2 r-3) / 3} T^{\prime}(r+2,2 j+1) x^{2 r-3 j-1}+\sum_{j=1}^{2 r / 3} T^{\prime}(r+2,2 j) x^{2 r-3 j+1} \\
x t_{r+1}^{\prime}(x)=T^{\prime}(r+1,0) x^{2 r-1}+\sum_{j=0}^{(2 r-6) / 3} T^{\prime}(r+1,2 j+1) x^{2 r-3 j-4}+\sum_{j=1}^{(2 r-3) / 3} T^{\prime}(r+1,2 j) x^{2 r-3 j-2}
\end{gathered}
$$

by taking the sums of both equality and simplified them, we get

$$
\begin{aligned}
\sum_{j=0}^{(2 r-3) / 3} T^{\prime}(r+2,2 j+1) x^{2 r-3 j-1} & =T^{\prime}(r+2,1) x^{2 r-1}+T^{\prime}(r+2,3) x^{2 r-4}+\sum_{j=0}^{(2 r-9) / 3} T^{\prime}(r+2,2 j+5) x^{2 r-3 j-7} \\
\sum_{j=1}^{2 r / 3} T^{\prime}(r+2,2 j) x^{2 r-3 j+1} & =T^{\prime}(r+2,2) x^{2 r-2}+T^{\prime}(r+2,4) x^{2 r-5}+\sum_{j=1}^{(2 r-6) / 3} T^{\prime}(r+2,2 j+4) x^{2 r-3 j-5} \\
\sum_{j=0}^{(2 r-6) / 3} T^{\prime}(r+1,2 j+1) x^{2 r-3 j-4} & =T^{\prime}(r+1,1) x^{2 r-4}+\sum_{j=0}^{(2 r-9) / 3} T^{\prime}(r+1,2 j+3) x^{2 r-3 j-7} \\
\sum_{j=1}^{(2 r-3) / 3} T^{\prime}(r+1,2 j) x^{2 r-3 j-2} & =T^{\prime}(r+1,2) x^{2 r-5}+\sum_{j=1}^{(2 r-6) / 3} T^{\prime}(r+1,2 j+2) x^{2 r-3 j-5}
\end{aligned}
$$

according to the definition of generalized Tribonacci polynomials (10), we have

$$
\begin{aligned}
x^{2} t_{r+2}^{\prime}(x) & +x t_{r+1}^{\prime}(x)+t_{r}^{\prime}(x) \\
= & T^{\prime}(r+2,0) x^{2 r+2}+\left[T^{\prime}(r+2,3)+T^{\prime}(r+1,1)+T^{\prime}(r, 0)\right] x^{2 r-4} \\
& +\left[T^{\prime}(r+1,0)+T^{\prime}(r+2,1)\right] x^{2 r-1} \\
& +\sum_{j=0}^{(2 r-9) / 3}\left[T^{\prime}(r+2,2 j+5)+T^{\prime}(r+1,2 j+3)+T^{\prime}(r, 2 j+1)\right] x^{2 r-3 j-7} \\
& +\left[T^{\prime}(r+2,4)+T^{\prime}(r+1,2)\right] x^{2 r-5} \\
& +\sum_{j=1}^{(2 r-6) / 3}\left[T^{\prime}(r+2,2 j+4)+T^{\prime}(r+1,2 j+2)+T^{\prime}(r, 2 j)\right] x^{2 r-3 j-5} \\
& +T^{\prime}(r+2,2) x^{2 r-2} .
\end{aligned}
$$


Since

$$
\begin{aligned}
T^{\prime}(r+3,3) & =T^{\prime}(r+2,3)+T^{\prime}(r+1,1)+T^{\prime}(r, 0), \\
\text { and } T^{\prime}(r+3,1) & =T^{\prime}(r+1,0)+T^{\prime}(r+2,1), \\
T^{\prime}(r+3,2 j+5) & =T^{\prime}(r+2,2 j+5)+T^{\prime}(r+1,2 j+3)+T^{\prime}(r, 2 j+1), \\
\text { and } T^{\prime}(r+2,0) & =T^{\prime}(r+3,0) \\
T^{\prime}(r+3,2 j+4) & =T^{\prime}(r+2,2 j+4)+T^{\prime}(r+1,2 j+2)+T^{\prime}(r, 2 j) \\
\text { and } T^{\prime}(r+3,2) & =T^{\prime}(r+2,2), \\
T^{\prime}(r+3,4) & =T^{\prime}(r+2,4)+T^{\prime}(r+1,2),
\end{aligned}
$$

then

$$
\begin{aligned}
x^{2} t_{r+2}^{\prime}(x)+x t_{r+1}^{\prime}(x)+t_{r}^{\prime}(x)= & T^{\prime}(r+3,0) x^{2 r+2}+T^{\prime}(r+3,3) x^{2 r-4} \\
& +T^{\prime}(r+3,1) x^{2 r-1}+T^{\prime}(r+3,2) x^{2 r-2}+T^{\prime}(r+3,4) x^{2 r-5} \\
& +\sum_{j=0}^{(2 r-9) / 3} T^{\prime}(r+3,2 j+5) x^{2 r-3 j-7} \\
& +\sum_{j=1}^{(2 r-6) / 3} T^{\prime}(r+3,2 j+4) x^{2 r-3 j-5}
\end{aligned}
$$

Also, we rewrite the sums in the right-hand side of the last equality, as follows

$$
\begin{aligned}
\sum_{j=0}^{(2 r-9) / 3} T^{\prime}(r+3,2 j+5) x^{2 r-3 j-7} & =\sum_{j=0}^{(2 r-3) / 3} T^{\prime}(r+3,2 j+1) x^{2 r-3 j-1} \\
& -T^{\prime}(r+3,1) x^{2 r-1}-T^{\prime}(r+3,3) x^{2 r-4} \\
\sum_{j=1}^{(2 r-6) / 3} T^{\prime}(r+3,2 j+4) x^{2 r-3 j-5} & =\sum_{j=1}^{(2 r) / 3} T^{\prime}(r+3,2 j) x^{2 r-3 j+1} \\
& -T^{\prime}(r+3,2) x^{2 r-2}-T^{\prime}(r+3,4) x^{2 r-5}
\end{aligned}
$$

Finally,

$$
\begin{aligned}
x^{2} t_{r+2}^{\prime}(x)+x t_{r+1}^{\prime}(x)+t_{r}^{\prime}(x)=T^{\prime}(r+3,0) & +\sum_{j=0}^{(2 r-3) / 3} T^{\prime}(r+3,2 j+1) x^{2 r-3 j-1} \\
& +\sum_{j=1}^{(2 r) / 3} T^{\prime}(r+3,2 j) x^{2 r-3 j+1} \\
& =t_{r+3}^{\prime}(x) .
\end{aligned}
$$

Thus, the formula is true for any positive integer $n \equiv 0[3]$. Analogously, we use the same technique with the other case $n \equiv 1[3]$ and $n \equiv 2[3]$ 
Theorem 3.3. For $n \geq 0$, we have

$$
t_{n}(x)=\frac{1}{\lambda}\left(\mathfrak{R}_{1}^{n+1}\left(\mathfrak{R}_{2}-\mathfrak{R}_{3}\right)+\mathfrak{R}_{2}^{n+1}\left(\mathfrak{R}_{3}-\mathfrak{R}_{1}\right)+\mathfrak{R}_{3}^{n+1}\left(\mathfrak{R}_{1}-\mathfrak{R}_{2}\right)\right),
$$

where $\lambda=\left(\mathfrak{R}_{1}-\mathfrak{R}_{2}\right)\left(\mathfrak{R}_{1}-\mathfrak{R}_{3}\right)\left(\mathfrak{R}_{2}-\mathfrak{R}_{3}\right)$ and $\mathfrak{R}_{1}, \mathfrak{R}_{2}, \mathfrak{R}_{3}$ are the distinct roots of the characteristic polynomial of (1).

Proof. Using the diagonalization of the generating matrix $Q$, so the characteristic equation of the generating matrix $Q$ is

$$
0=\left|Q-t I_{3}\right|=\left|\left(\begin{array}{ccc}
x^{2}-t & x & 1 \\
1 & -t & 0 \\
0 & 1 & -t
\end{array}\right)\right|=-t^{3}+x^{2} t^{2}+x t+1 .
$$

Let $\mathfrak{R}_{1}, \mathfrak{R}_{2}$ and $\mathfrak{R}_{3}$ be the roots of the characteristic equation $-t^{3}+x^{2} t^{2}+x t+1=0$ and also $\mathfrak{R}_{1}, \mathfrak{R}_{2}$ and $\mathfrak{R}_{3}$ be the three eigenvalues of the square matrix $Q$.

Let $P$ is a matrix of eigenvalues, we get

$$
P=\left(\begin{array}{ccc}
\mathfrak{R}_{1}^{2} & \mathfrak{R}_{2}^{2} & \mathfrak{R}_{3}^{2} \\
\mathfrak{R}_{1} & \mathfrak{R}_{2} & \mathfrak{R}_{3} \\
1 & 1 & 1
\end{array}\right)
$$

and

$$
P^{-1}=\frac{1}{\lambda}\left(\begin{array}{ccc}
\mathfrak{R}_{2}-\mathfrak{R}_{3} & \mathfrak{R}_{3}^{2}-\mathfrak{R}_{2}^{2} & \mathfrak{R}_{2} \mathfrak{R}_{3}\left(\mathfrak{R}_{2}-\mathfrak{R}_{3}\right) \\
\mathfrak{R}_{3}-\mathfrak{R}_{1} & \mathfrak{R}_{1}^{2}-\mathfrak{R}_{3}^{2} & \mathfrak{R}_{1} \mathfrak{R}_{3}\left(\mathfrak{R}_{3}-\mathfrak{R}_{1}\right) \\
\mathfrak{R}_{1}-\mathfrak{R}_{2} & \mathfrak{R}_{2}^{2}-\mathfrak{R}_{1}^{2} & \mathfrak{R}_{1} \mathfrak{R}_{2}\left(\mathfrak{R}_{1}-\mathfrak{R}_{2}\right)
\end{array}\right)
$$

where $\lambda=\left(\Re_{1}-\mathfrak{R}_{2}\right)\left(\Re_{1}-\mathfrak{R}_{3}\right)\left(\mathfrak{R}_{2}-\mathfrak{R}_{3}\right)$. Now, let

$$
D=\left(\begin{array}{lll}
\mathfrak{R}_{1} & 0 & 0 \\
0 & \mathfrak{R}_{2} & 0 \\
0 & 0 & \mathfrak{R}_{3}
\end{array}\right),
$$

i.e., $D$ is the diagonal matrix in which the eigenvalues of $Q$ are on the main diagonal. Then $Q=P D P^{-1}$, so we get

$$
\begin{aligned}
Q^{n} & =P D^{n} P^{-1} \\
& =\frac{1}{\lambda}\left(\begin{array}{ccc}
\mathfrak{R}_{1}^{n+2} & \mathfrak{R}_{2}^{n+2} & \mathfrak{R}_{3}^{n+2} \\
\mathfrak{R}_{1}^{n+1} & \mathfrak{R}_{2}^{n+1} & \mathfrak{R}_{3}^{n+1} \\
\mathfrak{R}_{1}^{n} & \mathfrak{R}_{2}^{n} & \mathfrak{R}_{3}^{n}
\end{array}\right)\left(\begin{array}{ccc}
\mathfrak{R}_{2}-\mathfrak{R}_{3} & \mathfrak{R}_{3}^{2}-\mathfrak{R}_{2}^{2} & \mathfrak{R}_{2} \mathfrak{R}_{3}\left(\mathfrak{R}_{2}-\mathfrak{R}_{3}\right) \\
\mathfrak{R}_{3}-\mathfrak{R}_{1} & \mathfrak{R}_{1}^{2}-\mathfrak{R}_{3}^{2} & \mathfrak{R}_{1} \mathfrak{R}_{3}\left(\mathfrak{R}_{3}-\mathfrak{R}_{1}\right) \\
\mathfrak{R}_{1}-\mathfrak{R}_{2} & \mathfrak{R}_{2}^{2}-\mathfrak{R}_{1}^{2} & \mathfrak{R}_{1} \mathfrak{R}_{2}\left(\mathfrak{R}_{1}-\mathfrak{R}_{2}\right)
\end{array}\right) .
\end{aligned}
$$

Finally

$$
t_{n}(x)=\frac{1}{\lambda}\left(\mathfrak{R}_{1}^{n+1}\left(\mathfrak{R}_{2}-\mathfrak{R}_{3}\right)+\mathfrak{R}_{2}^{n+1}\left(\mathfrak{R}_{3}-\mathfrak{R}_{1}\right)+\mathfrak{R}_{3}^{n+1}\left(\mathfrak{R}_{1}-\mathfrak{R}_{2}\right)\right)
$$




\subsection{Generating functions of the generalized Tribonacci sequence and polynomials}

Theorem 3.4. Let $T_{n}^{\prime}$ denote the $n$-th term of the generalized Tribonacci sequence, then the generating functions of the generalized Tribonacci sequence is given by

$$
T_{n}^{\prime}=\frac{\alpha^{n-3}\left((a+b) \alpha+b+c \alpha^{2}\right)}{(\alpha-\beta)(\alpha-\gamma)}+\frac{\beta^{n-3}\left((a+b) \beta+b+c \beta^{2}\right)}{(\beta-\alpha)(\beta-\gamma)}+\frac{\gamma^{n-3}\left((a+b) \gamma+b+c \gamma^{2}\right)}{(\gamma-\alpha)(\gamma-\beta)} .
$$

Proof. By using the Theorem 2.1 and the generating function of the Tribonacci sequence (3). We get the results.

Theorem 3.5. The $n$-th generalized Tribonacci polynomials is given by

$$
t_{n}^{\prime}(x)=\frac{\mathfrak{R}_{1}^{n-3}\left((a+b x) \mathfrak{R}_{1}+b+c \Re_{1}^{2}\right)}{\left(\mathfrak{R}_{1}-\mathfrak{R}_{2}\right)\left(\mathfrak{R}_{1}-\mathfrak{R}_{3}\right)}+\frac{\mathfrak{R}_{2}^{n-3}\left((a+b x) \mathfrak{R}_{2}+b+c \mathfrak{R}_{2}^{2}\right)}{\left(\mathfrak{R}_{2}-\mathfrak{R}_{1}\right)\left(\mathfrak{R}_{2}-\mathfrak{R}_{3}\right)}+\frac{\mathfrak{R}_{3}^{n-3}\left((a+b x) \mathfrak{R}_{3}+b+c \mathfrak{R}_{3}^{2}\right)}{\left(\mathfrak{R}_{3}-\mathfrak{R}_{1}\right)\left(\mathfrak{R}_{3}-\mathfrak{R}_{2}\right)},
$$

where $\mathfrak{R}_{1}, \mathfrak{R}_{2}$ and $\mathfrak{R}_{3}$ are the distinct roots of the equation (5).

Proof. Using the relation between Tribonacci polynomials and generalized Tribonacci polynomials (Theorem 3.1) and the formula of Tribonacci polynomial (Eq (4)).

Proposition 3.3. For $n \geqslant 4$, we have

$$
\mathfrak{R}^{n}=t_{n-1}(x) \mathfrak{R}^{2}+\left[x t_{n-2}(x)+t_{n-3}(x)\right] \mathfrak{R}+t_{n-2}(x)
$$

where $\mathfrak{R}$ stands for a root of the equation (5).

Proof. By induction on $n$.

Proposition 3.4. For any integer $n \geq 3$

$$
\left(\mathfrak{R}_{1}^{n}+\mathfrak{R}_{2}^{n}+\mathfrak{R}_{3}^{n}\right)=t_{n+1}(x)+x t_{n-1}(x)+2 t_{n-2}(x)
$$

Theorem 3.6. For $n \geq 1$, we have

$$
t_{3 n-1}(x)=\sum_{i=0}^{n} \sum_{j=0}^{i}\left(\begin{array}{c}
n \\
i
\end{array}\right)\left(\begin{array}{l}
i \\
j
\end{array}\right) x^{i+j} t_{i+j-1}(x) .
$$

Proof. Let $\mathfrak{R}$ stand for a root of the equation (5). Then

$$
\begin{aligned}
\left(\mathfrak{R}^{3}\right)^{n} & =\sum_{i=0}^{n}\left(\begin{array}{c}
n \\
i
\end{array}\right)\left(x^{2} \mathfrak{R}^{2}+x \mathfrak{R}\right)^{i} \\
& =\sum_{i=0}^{n} \sum_{j=0}^{i}\left(\begin{array}{c}
n \\
i
\end{array}\right)\left(\begin{array}{c}
i \\
j
\end{array}\right)(x)^{i+j} \mathfrak{R}^{i+j} .
\end{aligned}
$$

If we replace $\mathfrak{R}$ to $\mathfrak{R}_{1}, \mathfrak{R}_{2}, \mathfrak{R}_{3}$, and using the equation (5), then we obtain

$$
\begin{aligned}
t_{3 n-1}(x) & =\sum_{i=0}^{n} \sum_{j=0}^{i}\left(\begin{array}{c}
n \\
i
\end{array}\right)\left(\begin{array}{c}
i \\
j
\end{array}\right) x^{i+j}\left(\frac{\mathfrak{R}_{1}^{i+j}}{\left(\mathfrak{R}_{1}-\mathfrak{R}_{2}\right)\left(\mathfrak{R}_{1}-\mathfrak{R}_{3}\right)}+\frac{\mathfrak{R}_{2}^{i+j}}{\left(\mathfrak{R}_{2}-\mathfrak{R}_{1}\right)\left(\mathfrak{R}_{2}-\mathfrak{R}_{3}\right)}+\frac{\mathfrak{R}_{3}^{i+j}}{\left(\mathfrak{R}_{3}-\mathfrak{R}_{1}\right)\left(\mathfrak{R}_{3}-\mathfrak{R}_{2}\right)}\right) \\
& =\sum_{i=0}^{n} \sum_{j=0}^{i}\left(\begin{array}{c}
n \\
i
\end{array}\right)\left(\begin{array}{c}
i \\
j
\end{array}\right)(x)^{i+j} t_{i+j-1}(x) .
\end{aligned}
$$




\subsection{Generating matrix of the Tricobsthal polynomials}

The generating matrix of the Tricobsthal polynomial are introducing by $Q^{\prime}$

$$
Q^{\prime}=\left[\begin{array}{ccc}
1 & x & x^{2} \\
1 & 0 & 0 \\
0 & 1 & 0
\end{array}\right]
$$

We can prove that by induction on $n$ :

$$
\left(Q^{\prime}\right)^{n}=\left(\begin{array}{ccc}
1 & x & x^{2} \\
1 & 0 & 0 \\
0 & 1 & 0
\end{array}\right)^{n}=\left(\begin{array}{ccc}
J_{n+1}(x) & J_{n+2}(x)-J_{n+1}(x) & x^{2} J_{n}(x) \\
J_{n}(x) & J_{n+1}(x)-J_{n}(x) & x^{2} J_{n-1}(x) \\
J_{n-1}(x) & J_{n}(x)-J_{n-1}(x) & x^{2} J_{n-2}(x)
\end{array}\right) .
$$

Theorem 3.7. For $n \geq 4$, we have

$$
J_{n}^{\prime}(x)=\left(c_{1} x+c_{0}\right) J_{n-2}(x)+b\left[J_{n-1}(x)-J_{n-2}(x)\right]+a x^{2} J_{n-3}(x),
$$

where $c_{1} \geq 0$ and $a, b, c_{0}$ are non-negative integers.

Proof. Using the same technique as in Theorem 3.1.

Theorem 3.8. For $n \geq 0$, we have

$$
J_{n}(x)=\frac{1}{\lambda}\left[\left(\mathfrak{R}_{1}^{\prime}\right)^{n+1}\left(\mathfrak{R}_{2}^{\prime}-\mathfrak{R}_{3}^{\prime}\right)+\left(\mathfrak{R}_{2}^{\prime}\right)^{n+1}\left(\mathfrak{R}_{3}^{\prime}-\mathfrak{R}_{1}^{\prime}\right)+\left(\mathfrak{R}_{3}^{\prime}\right)^{n+1}\left(\mathfrak{R}_{1}^{\prime}-\mathfrak{R}_{2}^{\prime}\right)\right],
$$

where $\lambda^{\prime}=\left(\mathfrak{R}_{1}^{\prime}-\mathfrak{R}_{2}^{\prime}\right)\left(\mathfrak{R}_{1}^{\prime}-\mathfrak{R}_{3}^{\prime}\right)\left(\mathfrak{R}_{2}^{\prime}-\mathfrak{R}_{3}^{\prime}\right)$ and $\mathfrak{R}_{1}^{\prime}, \mathfrak{R}_{2}^{\prime}, \mathfrak{R}_{3}^{\prime}$ are the distinct roots of the characteristic polynomial of (6)

$$
t^{3}-t^{2}-x t-x^{2}=0
$$

Proof. Using the same technique as in Theorem 3.3.

Proposition 3.5. For any integer $n \geq 3$

$$
\left(\mathfrak{R}_{1}^{\prime n}+\mathfrak{R}_{2}^{\prime n}+\mathfrak{R}_{3}^{\prime n}\right)=J_{n+1}(x)+x J_{n-1}(x)+2 x^{2} J_{n-2}(x),
$$

where $\mathfrak{R}_{1}^{\prime}, \mathfrak{R}_{2}^{\prime}$ and $\mathfrak{R}_{3}^{\prime}$ are the distinct roots of the equation (9)

Proposition 3.6. For $n \geqslant 3$, we have

$$
\mathfrak{R}^{\prime n}=J_{n-1}(x) \mathfrak{R}^{\prime 2}+\left[J_{n}(x)-J_{n-1}(x)\right] \mathfrak{R}^{\prime}+x^{2} J_{n-2}(x),
$$

where $\mathfrak{R}^{\prime}$ stands for a root of the equation (8).

Proof. The proof of this proposition doing by induction on $n$.

Theorem 3.9. For $n \geq 1$, we have

$$
J_{3 n-1}(x)=\sum_{i=0}^{n} \sum_{k=0}^{i}\left(\begin{array}{c}
n \\
i
\end{array}\right)\left(\begin{array}{l}
i \\
k
\end{array}\right)(x)^{2 n-i-k} J_{i+k-1}(x) .
$$

Proof. Using the same technique as in Theorem 3.6. 


\section{Acknowledgements}

We would like to thank an anonymous referees for reading this article and the suggestions given.

\section{References}

[1] Badidja, S., \& Boudaoud, A. (2017). A unique representation of positive integers as a sum of distinct Tribonacci numbers. Journal of Mathematics and Statistics, 13(1), 57-61.

[2] Badidja, S. (2018). Dèecomposition d'entiers illimitès et des termes d'ordre illimitès des suites recurrences linèaires. $\mathrm{PhD}$ thesis, University of Mohamed Boudiaf, Msila, Algeria.

[3] Chelgham, M., \& Boussayoud, A. (2020). Construction of symmetric functions of generalized Tribonacci numbers. Journal of Science and Arts, 1(50), 65-74.

[4] Gupta, Y. K., Badshah, V. H., Singh, M., \& Sisodiya, K. (2016). Some identities of Tribonacci polynomials. Turkish Journal of Analysis and Number Theory, 4(1), 20-22.

[5] Koshy, T. (2001). Fibonacci and Lucas Numbers with Applications, Wiley-Interscience Publication.

[6] Panwar, Y. K., Singh B., \& Gupta V. K. (2013). Generalized Fibonacci polynomials. Turkish Journal of Analysis and Number Theory, 1(1), 43-47.

[7] Rybołowicz, B., \& Tereszkiewicz, A. (2018). Generalized Tricobsthal and generalized Tribonacci polynomials. Applied Mathematics and Computation, 325, 297-308.

[8] Soykan, Y., \& Okumus, I. (2019). On a generalized Tribonacci sequence. Journal of Progressive Research in Mathematics, 14(3), 1-9. 